\title{
Diosgenin induces apoptosis in HepG2 cell through an ASK1-ROS-p38 MAPK/JNK cascade
}

\author{
D. S. Kim ${ }^{1}$, B. K. Jeon ${ }^{1}$, S. Sin ${ }^{2}$, Y. J. Mun ${ }^{3}$, W. H. Woo ${ }^{3}$ and Y. E. Lee ${ }^{2}$ \\ ${ }^{1}$ Department of Herbal Resources, Professional Graduate School of Oriental Medicine, ${ }^{2}$ Dept of Food and Nutrition, \\ College of Human Environmental Resources, Wonkwang University, Iksan, Republic of Korea and ${ }^{3}$ Department of Anatomy, \\ College of Oriental Medicine
}

Food saponins have been used in complimentary and traditional medicine against a variety of diseases including several cancers. Diosgenin, a naturally occurring steroid saponin found abundantly in legumes and yams, has been shown to have antitumor effects on cancer cells $^{(1)}$. Apoptosis signal-regulating kinase (ASK)-1, as a mitogen-activated protein kinase kinase kinase (MAPKKK), has been implicated in cytokine- and stress-induced apoptosis ${ }^{(2)}$. To elucidate the cytotoxicity mechanism of diosgenin, we investigated the role of ASK-1-p38 MAPK/JNK cascade in apoptosis and caspase-3 activation in diosgenin-treated HepG2 cells.

HepG 2 cells were treated with different doses of diosgenin $(0 \sim 40 \mu \mathrm{M}, n 3-5$ per group $)$ for $24 \mathrm{~h}$ at $37^{\circ} \mathrm{C}$. Phosphorylated MAPK, ASK1 and caspase levels were detected by Western blotting and reactive oxygen species (ROS) production was detected by fluorescence microscopy. Results were obtained from three independent experiments.

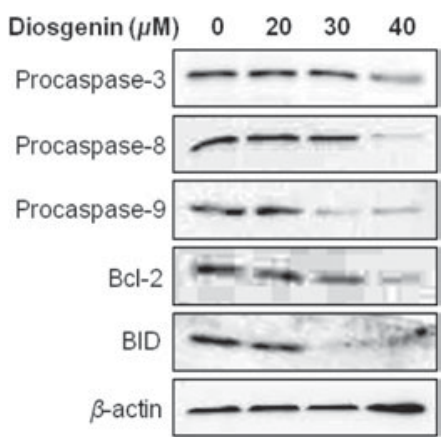

Fig. 1. Caspase, antiapoptotic protein.

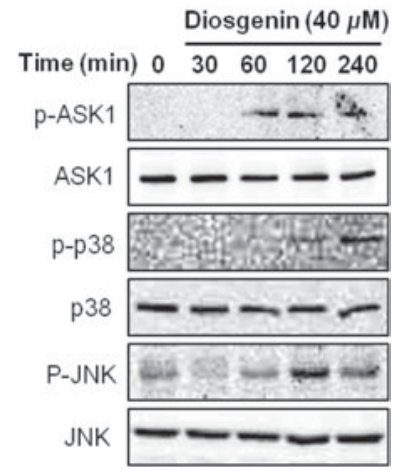

Fig. 2. ASK-1, p38 and JNK activation.

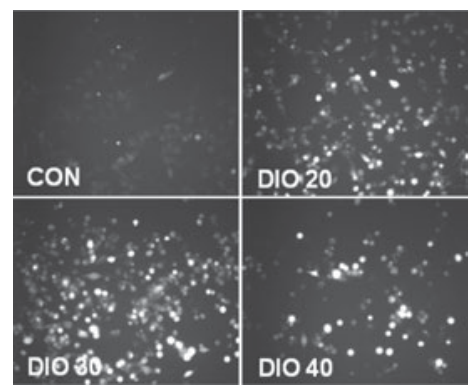

Fig. 3. ROS production.

The results showed that the induction of apoptosis in HepG2 cells by diosgenin was characterised by DNA fragmentation, the activation of caspase- 3 and the cleavage of poly ADP-ribose polymerase (PARP). The expressions of Bcl-2 protein and Bid protein were reduced by diosgenin, whereas Bax protein and the release of Cytochrome c were increased in the cytosol (Fig. 1). Diosgenin induced ASK-1 phosphorylation and concomitantly p38 MAPK and c-Jun N-terminal kinase (JNK) phosphorylation as well as induced caspase-3 and - 9 in HepG2 cells (Fig. 2). Furthermore diosgenin-induced apoptosis was preceded by increased generation of ROS (Fig. 3). In conclusion, these results indicated that the treatment of HepG2 cells with diosgenin triggers generation of ROS, resulting in dissociation of the ASK1mediated activation of JNK and p38 pathways.

This work was supported by the Ministry of Knowledge Economy, Republic of Korea 2009.

1. Dhingra N, Bhardwaj TR, Mehta N et al. (2010) Eur J Med Chem 45, 2229-2236.

2. Machino T, Hashimoto S, Maruoka S et al. (2003) Crit Care Med 31, 2776-2781.

3. Lepage C, Leger DY, Bertrand J et al. (2010) Cancer Lett 301, 193-202. 